\title{
EL DOBLE DESCUBRIMIENTO EN RAPA NUI: UNA MIRADA DESDE LOS TEXTILES
}

\author{
The Double Discovery on Rapa Nui: A Textile Perspective
}

\section{ANDREA SEELENFREUND* \& ANTONIA MARDONES**}

Fecha de recepción: 06 de julio de 2018 - Fecha de aprobación: 10 de diciembre de 2018

\section{Resumen}

Analizamos algunos aspectos de los intercambios materiales y el rol de los textiles en las relaciones entre los nativos de Rapa Nui y los europeos de las tres primeras expediciones que la visitaron en el siglo XVIII. Buscamos entender el rol de los textiles y las vestimentas al interior de la cultura rapanui analizando el contexto y las creencias en las que estos se insertan, y en particular el rol de las telas de corteza batanada (o tapa) y los textiles europeos, intercambiados en esas ocasiones. El objetivo del análisis es comprender estos primeros encuentros por medio de la agencia de un elemento de la cultura material y desde de la perspectiva indígena. Demostramos que las formas de entender y utilizar el textil corresponden a una estructura simbólicamente significativa y compartida con el resto de la Polinesia, que les permitió articular lo autóctono con lo extranjero y regular las fronteras en una situación de contacto cultural.

Palabras clave: Isla de Pascua; primeros encuentros; intercambio de dones; textiles de corteza; tapa.

\section{Abstract}

In this paper we analyze some aspects of the material exchanges concerning cloth and clothing between the Rapanui people and Europeans as disclosed in the accounts and log books of the first three European expeditions that visited the island in the 18th Century. We consider the context of social organization and beliefs in which textiles, particularly beaten bark cloth textiles (tapa) and European cloth were given at the time of these encounters. The aim of the analysis is to understand these first encounters through the agency of a particular material culture, an exercise into indigenous insights that has rarely been attempted for this island in the Pacific. We show that the structure in which textiles were understood and used was shared with other Polynesian islands. Textiles were key symbolic objects in this situation of cultural contact, regulating and articulating cultural borders.

Keywords: Easter Island; first encounters; gift exchange; bark cloth textiles; tapa.

* Dra. en Arqueología. Académica de la Escuela de Antropología, Universidad Academia de Humanismo Cristiano, Santiago, Chile. El artículo está enmarcado en los proyectos Fondecyt 1120175 y 1180052. Correo-e: aseelenfreund@academia.cl, aseelenfreund@gmail.com

** Lcda. en Antropología. Escuela de Antropología, Universidad Academia de Humanismo Cristiano, Santiago, Chile. Correo-e: amardones@gmail.com 


\section{Introducción}

En los encuentros ocurridos entre europeos e isleños en Oceanía durante los siglos XVII, XVIII y XIX se produjo un fenómeno de contacto entre dos mundos que intentaron comprenderse mutuamente. Esto generó procesos de incorporación de la alteridad en ambos lados de la playa (Dening, 1980) y donde el "otro" fue entendido desde la ontología propia de cada una de las sociedades involucradas. Los estudios históricos y antropológicos sobre esta materia se han centrado en el recibimiento dado a los europeos por parte de los isleños y las percepciones mutuas que se generaron, así como en los malentendidos o las interpretaciones que dieron los europeos a las prácticas isleñas, y también en las miradas muchas veces contrapuestas que los polinésicos tuvieron sobre los mismos acontecimientos (Pearson, 1969; Howe, 1977; Campbell, 1982, 2003; McLachlan, 1982; Quanchi \& Adams, 1993; Sahlins, 1997; Tcherkézoff, 2003, 2012; Jolly y Tcherkézoff, 2009, entre otros).

El fenómeno del encuentro generó, en ambas partes, la necesidad de posicionar a la otredad en una categoría nueva o de adaptar las categorías preexistentes en una estructura significativa (Campbell, 1997; Tcherkézoff, 2004). Así como los viajeros observaron a los isleños desde los imaginarios de la Europa de la época, los isleños, a su vez, percibieron a los europeos como seres no pertenecientes al mundo de lo humano polinésico. Como señalan Sahlins (1997) y Tcherkézoff (2004), si bien las sociedades del Pacífico no estaban confinadas a las islas en que residían y mantenían contacto marítimo con el exterior, "los polinésicos solo conocían hombres como ellos mismos" (Tcherkézoff, 2004, p. 137).
Según Campbell (1997), el fenómeno del encuentro cultural con navegantes europeos en el Pacífico generó transformaciones en la ontología polinésica. Las estructuras significativas, e incluso la mitología, tuvieron que adecuarse para que estos humanos (o seres) de otro tipo fueran incorporados y registrados dentro del mundo conocido por los isleños. En ocasiones adoptaron un modelo de alteridad que les permitió la integración de la diferencia, lo que generó transformaciones a nivel cosmológico (Tcherkézoff, 2004).

En su clásico estudio sobre la visita del capitán James Cook a Hawái, Sahlins (1997) señala que en dicho encuentro los isleños vieron a Cook como una manifestación del dios local Lono, pues sus barcos habían llegado a las costas desde el este, de donde proviene la luz del sol, justo en la época en que se realizaba el ritual de renovación, en el que dicha divinidad era representada para cerrar un ciclo y abrir otro nuevo. Sin embargo, es importante de visualizar que los polinésicos no entendieron a los europeos como dioses u hombres bajo la concepción judeocristiana, que los percibe como conceptos opuestos excluyentes, sino como manifestación de lo sagrado, manifestación que era parte de un todo integrado. Dentro de la ontología polinésica, la divinidad tenía relación con la integración del todo (el cosmos) con sus diferentes partes. Así como lo divino era un todo invisible que se manifestaba parcialmente en personas y objetos, el jefe era una parte de ese todo, una manifestación visible de la divinidad. En esta manera de entender el mundo, los "visitantes" extranjeros que llegaron a las costas podían ser expresiones de esa totalidad (Tcherkézoff, 2004; Jolly \& Tcherkézoff, 2009). 
Varios elementos propios de la concepción de mundo polinésica fundamentan esta visión de los europeos. En primera instancia, los isleños prestaron atención a la blancura de las pieles de los extranjeros, blancura que se asociaba a las características sagradas de la luminosidad del sol, que hace visible el mundo. Además, los navegantes europeos traían consigo varios artefactos que también fueron asociados a aspectos suprahumanos, pues encajaban en el lugar privilegiado que se le otorgaba a la luminosidad: sus armas de fuego, sus ornamentos de vidrio y metal que reflejaban la luz del sol y, finalmente, sus ropas (Sahlins, 1997; Tcherkézoff, 2004).

Dentro de la ontología polinésica, a los extranjeros que venían del otro lado del mar se les otorgaba un estatus de divinidad semejantes a la de los altos jefes polinésicos, quienes poseían un carácter sagrado y eran considerados como extraños o invasores, como seres de origen extralocal al estar cercanos a las divinidades (Thomas, 1991). La razón de esto la encontramos en los mitos de origen y en la concepción polinésica del espacio y el tiempo. Como indica Tcherkézoff (2004), en la cosmovisión polinésica el espacio se funde en forma indisoluble con el tiempo. El mundo era un círculo plano de islas rodeadas de mar y cubiertas por el cielo, que en forma de campana se extendía sobre las islas y el mar. Tanto el pasado como el presente se desarrollaban bajo este cielo acampanado. Según el mito, la vida solo se hizo posible gracias a un héroe mítico que levantó ese cielo y generó con ello la luz y la vida. Los antepasados deificados de cada clan llegaron en sus islas barcos a habitar los mares y sus islas. La isla que estaba más distante en el espacio era siempre aquella que podía estar más próxima en el tiempo al punto de origen donde comenzó el mundo de la luz. En esta visión, la historia de cada isla siempre estaba ligada a un barco/isla de origen. Así, el punto de salida/origen de cada barco representaba el punto de inicio de cada genealogía. La división territorial de una isla se organizaba jerárquicamente de acuerdo con el tiempo de ocupación desde el momento del asentamiento inicial por un grupo de parentesco. De este modo, la organización espacial era un espejo histórico del tiempo transcurrido desde ese momento de origen. En este escenario, los nuevos visitantes fueron incorporados dentro de la estructura significativa polinésica como seres vinculados con lo sagrado que venían de un espacio más cercano al origen. Las acciones de los isleños estuvieron orientadas a domesticar a los extranjeros para poder incorporar sus poderes o capacidades de controlar cosas que ellos no conocían.

Varios autores (Colchester, 2003; Küchler, 2003; Jolly, 2014, entre otros) han relevado la importancia que ha tenido el textil en la historia de las sociedades isleñas y su contacto con Occidente. Sin embargo, el fenómeno del textil no ha sido trabajado en el abordaje que la antropología histórica ha hecho del contacto cultural para el caso rapanui. En el presente artículo se busca indagar en esta relación a partir de un análisis de los datos entregados por las crónicas de viajes de las tres primeras expediciones que visitaron Rapa Nui en el siglo XVIII. Intentaremos separar las descripciones de las interpretaciones realizadas por los mismos navegantes sobre los eventos vividos. Este primer análisis se complementará con un análisis etnográfico derivado de algunas observaciones y visitas que datan de periodos posteriores, tanto en Rapa Nui como en otras islas del Pacífico. 


\section{Textil en los primeros encuentros en Polinesia}

Tcherkézoff (2003) señala que, dentro del intercambio cultural producido durante el contacto, las telas jugaron un importante papel en la forma en que ambas sociedades se comprendieron mutuamente. El punto de unión entre las dos culturas era el hecho de que tanto para los europeos como para los polinésicos, cubrirse el cuerpo con capas de tela constituía un signo de estatus. De este modo, ambos grupos reconocían a las jefaturas de los otros: al capitán, que poseía un uniforme diferenciado del resto, y al jefe, cuyo cuerpo estaba cubierto de telas de corteza, denominadas tapa, y esteras tejidas.

La interpretación errada de los europeos se dio, en primera instancia, por considerar que la vestimenta podía variar solo en términos de la técnica o del diseño de las confecciones y no en términos de su significado dentro de las relaciones sociales, sin darse cuenta de que en la Polinesia ciertos tipos de vestimenta eran objetos de gran valor ceremonial. Malinterpretaron la utilización y la manipulación social de las telas, viendo en el acto de envolver y desenvolver en tapa solo una acción de vestir y desvestir desde la concepción occidental judeocristiana, basada en categorías opuestas y excluyentes, donde lo vestido se opone a lo desvestido, haciendo un vínculo directo entre desnudez y deseo sexual (Tcherkézoff, 2003).

Lo que los europeos ignoraban era que la cultura polinésica tenía una forma particular de entender los regalos en telas de corteza. Cuando un extranjero arribaba a una isla, se ofrecía a quedar bajo el mando del jefe local, para lo cual llegaba con comida. En respuesta a este gesto, los residentes le entregaban un regalo en tela de tapa. El acto ritual de bienvenida tenía un significado doble: al presentarse ataviado con sus más preciadas pertenencias, el anfitrión daba cuenta de que consideraba al extraño como superior. Al mismo tiempo, el acto de envolver en tapa era también una forma de incorporar al extraño y domesticar sus poderes.

Estos ritos fueron interpretados por los europeos como actos de provocación sexual, particularmente cuando las personas que se desenvolvían en metros de capas de telas eran mujeres muy jóvenes. En Polinesia, hasta hace poco, la forma más respetuosa de presentar un regalo de telas era que lo llevara una mujer envuelta en ella, la cual se desenvolvía frente al receptor hasta desnudar la parte superior del cuerpo. El acto de desnudarse era una señal de respeto frente a un extraño (Tcherkézoff, 2003; Jolly \& Tcherkézoff, 2009).

Los europeos vieron, entonces, en esta "desnudez" femenina una conducta lasciva, cuando en realidad dicha desnudez significaba un acto de respeto y sumisión. Esta percepción europea se liga además con la interpretación de las conductas femeninas como hospitalidad sexual, pues las jóvenes isleñas estaban constantemente invitando a los marineros a tener relaciones sexuales con ellas. Sin embargo, el hecho de que los jefes tahitianos, samoanos, hawaianos y también rapanui incitaran a las mujeres, sobre todo a las vírgenes, a tener relaciones sexuales con los europeos era para capturar el poder (mana) de los visitantes por medio del establecimiento metafórico de un matrimonio sagrado, domesticando así la potencia de los extranjeros a través de la descendencia común. 
Otra de las consecuencias o resultados de los primeros encuentros entre europeos y polinésicos fue que los primeros transformaron un acto ritual de bienvenida en uno de comercio. En una primera instancia los isleños no esperaban nada a cambio de los regalos sagrados. Sin embargo, al entregarles los europeos telas en retribución a los objetos, los polinésicos comenzaron a exigir dichas telas o a apoderarse de manteles, chaquetas, pañuelos, etc., en las expediciones posteriores (Jolly \& Tcherkézoff, 2009).

\section{Los textiles como objetos de intercambio}

En Polinesia, un objeto como un manto de tela de corteza puede mantener el rango, la autoridad y el poder de un jefe y enfatizar su poder divino, pues simbólicamente representa el origen mítico del grupo. A su vez, el mana ${ }^{1}$ se transmite a las mujeres en el proceso de fabricación de la tela, quienes en todo proceso creativo, tales como en el dar a luz o en la creación de un trozo de tela, entran en y participan de un estado sagrado. Los objetos que se fabrican también son tapu ${ }^{2}$ y si no se manejan de acuerdo con los parámetros correctos, tanto el artesano como quien lo use puede enfermarse o incluso morir.

En las sociedades polinésicas hay dos categorías de cosas involucradas en todo el acto social del don. Una está constituida por los alimentos y la otra por las "vestiduras sagradas". Una característica que distingue a las dos categorías es el carácter profano o sagrado del objeto dado. Según Tcherkézoff (2003), lo sagrado debe ser entendido como un elemento que simboliza la totalidad, es decir, el grupo en su conjunto, ya sea toda la sociedad o uno de sus subgrupos, como las familias, los linajes o los clanes. Solo los textiles tienen este carácter de sacralidad en la Polinesia y son, por lo tanto, los objetos de intercambio ritual por excelencia. Las telas de corteza, las esteras y las mantas, al pertenecer al grupo mayor, no son propiedad de un individuo, tienen inscritas la historia familiar, por tanto, a diferencia de los alimentos, están destinadas a circular. Otro elemento clave de los textiles es su vínculo con lo sagrado, su capacidad para atraer y captar la esencia de los dioses, característica que se hace presente en el acto ritual de envolver en telas (Tcherkézoff, 2003). El principal atributo de las telas sagradas (tapa) en la Polinesia es que dotan a la gente de la capacidad para capturar lo sagrado (el mana de los ancestros) por medio del acto ritual de descubrirse y cubrirse. El significado ritual de la tela de corteza se asocia con la piel y muchos de los rituales presentes en la Polinesia se estructuran a partir del acto de envolver y desenvolver. Así, tanto en los ritos funerarios como en la envestidura de un jefe se envuelve el cuerpo para atraer el mana de los ancestros o los dioses. El textil, además, también da cuenta de la sacralidad de los sitios, los restos mortuorios o las personas de alto rango social, que denotan su carácter tabú a través del acto de envolver (Babadzan, 2003; Thomas, 2003).

Dentro del ordenamiento social polinésico, las telas jugaban un rol fundamental, tanto en lo que respecta al ámbito político como a los aspectos ligados con la cosmovisión y sus manifestaciones rituales. Ciertos tipos de vestimenta eran considerados objetos sagrados, es decir, taonga, y, por ende, representaban la conexión con los ancestros-dioses. Se convertían así en los puntos de unión entre los grupos de parentesco, sus genealogías y tierras. Las telas eran un "tesoro trascendente", un documento histórico que autentificaba los 
poderes asociados con los ancestros (Weiner, 1992; Tcherkézoff, 2003).

Según Weiner (1992), estas telas, al ser consideradas como taonga y estar imbuidas de la identidad y la historia del grupo, eran apreciadas como posesiones inalienables, lo cual implicaba que se mantenían dentro de la familia: la pérdida de una de ellas implicaba la disminución del poder del grupo. Al estar imbuidas del mana de los ancestros, como ya se dijo, las capas de tela de corteza que pertenecían a los jefes daban cuenta de su autoridad y estatus. De este modo, existían posesiones que se intercambiaban y otras que se mantenían pues otorgaban estatus y daban cuenta de la jerarquía entre los diferentes grupos. Aunque los individuos o grupos de individuos intercambiaran en diferentes niveles, en cada intercambio la posesión de bienes inalienables marcaba una diferencia entre las partes que intercambiaban. Tapsell (1997) hace una corrección sobre los planteamientos de Weiner señalando que los taonga no pueden ser entendidos como posesiones. Los hombres no pueden "apropiarse" de un taonga pues estos son sus ancestros; pueden convertirse en sus protectores y cuidadores, pero no en sus dueños, pues la persona es la que pertenece al taonga (ancestro), no al revés.

En las sociedades polinésicas, el establecimiento de lazos políticos y económicos a partir del acto de intercambiar era primordial. El hecho de poseer bienes para el intercambio otorgaba estatus a los diferentes grupos. Ahora bien, el poder y la influencia de un jefe se manifestaba en la capacidad de expandir la identidad del grupo y esto era posible en la medida en que se era lo suficientemente rico como para capturar un taonga de otro grupo y tener acceso, de este modo, al poder de sus ancestros (Weiner, 1992).

El carácter sagrado de las telas les da el poder de incorporar a los ancestros a través del ritual y el intercambio (Tcherkézoff, 2012). Textiles y esculturas son los puntos de conexión entre grupos de parentesco, sus genealogías y la tierra, en cuanto representaciones espirituales de los ancestros. En este contexto cultural, los textiles son los objetos materiales por excelencia destinados para el intercambio. Las telas, como elemento material, servían para establecer fronteras simbólicas y sociales y, como tales, permiten manejar el tabú (Tcherkézoff, 2012). Los regalos de tela buscaban establecer una relación que no se basara en la violencia sino en el respeto y transformar una "forma externa de sacralidad que era peligrosa al ser tocada" (tapu, sa, ra'a, moa) en algo "tocable" (noa) (Tcherkézoff, 2008).

En estos primeros contactos y encuentros entre polinesios y europeos, los textiles participaron en las interacciones y, cómo no, también en los diversos malentendidos entre los dos sistemas mundo.

\section{Los primeros encuentros y el textil en Rapa Nui}

Los primeros encuentros son testimonio del vínculo que se estableció entre los rapanui y los europeos del siglo XVIII. McCall (1990) plantea que la experiencia con la primera expedición holandesa va a modelar la forma en que los rapanui se relacionarán con los extranjeros y posibilitar, a partir de esta, el establecimiento del comercio en especies de alimentos y el ofrecimiento de los favores sexuales de las mujeres como una de las características de dichas 
relaciones. Foerster (2012), por su parte, afirma que el "misterio" sobre Rapa Nui se construyó a partir de estos primeros encuentros, producto de las narrativas europeas que centraban su interés en las estatuas monumentales más que en los habitantes de las islas y sus costumbres, resaltando una imagen negativa y empobrecida de un pueblo con escasas riquezas para entregar al forastero.

En las narrativas europeas existen distintas aproximaciones positivas y negativas sobre los isleños según las diferentes exploraciones y las versiones de los tripulantes dentro de una misma expedición. Sin embargo, todos los consideran dentro de un estado inferior de desarrollo en comparación con la sociedad occidental.

A diferencia de los encuentros en el resto de la Polinesia, el contacto de los rapanui con los holandeses en 1722 puede haber sido el "primero" después de un largo tiempo y, por ende, de varias generaciones. Decimos el primero, pues sin los materiales para construir grandes canoas, el aislamiento de los rapanui se había intensificado drásticamente al quedar imposibilitados para navegar hacia otras islas. Lo cierto es que en Rapa Nui los contactos con otras poblaciones polinésicas no eran un fenómeno recurrente como entre otras islas del Pacífico durante la misma época. La pregunta es, entonces, cómo fueron considerados estos encuentros por los propios isleños y cómo fueron incorporados estos elementos ajenos en sus categorías y experiencias anteriores al contacto.

En este trabajo nos centraremos en la forma en que los rapanui se acercaron a este encuentro y cómo fueron incorporados los "nuevos visitantes" a la estructura significativa rapanui. Postulamos que la tesis planteada por Campbell (1982), Tcherkézoff (2003) y Sahlins (1997) para otras islas de la Polinesia también es válida para Rapa Nui, donde sus habitantes, en un comienzo, pueden haber considerado también a los europeos como manifestaciones del todo divino invisible. Sin embargo, al igual que Foerster (2012), creemos que cada una de las experiencias fue moldeando la relación con el barco siguiente y que los rapanui fueron acomodando su comportamiento durante el contacto inicial en las visitas subsiguientes.

\section{La expedición holandesa}

Los primeros europeos en llegar a Rapa Nui fueron los holandeses, quienes arribaron a las costas isleñas el 5 de abril de 1722 en una expedición compuesta por tres navíos con 271 tripulantes y 78 cañones, todos bajo la comandancia de Jacob Roggeveen.

Se puede inferir el impacto visual que debe haber causado la llegada de estas naves con altos mástiles de madera envueltos en telas blancas y coronados con banderas y banderines de colores (rojo, blanco y azulino, los colores de la armada holandesa de la época) en una isla desprovista, desde hacía al menos cincuenta años, de grandes árboles, según lo atestigua la evidencia arqueológica (Flenley, 1996; Flenley et al., 1991; Horrocks et al., 2012).

El desembarco fue un acto formal, de un pequeño cuerpo de hombres portando estandartes con banderines multicolores y vistiendo el uniforme de la Armada holandesa. Este constaba de chaquetas azulinas, vestones rojos y pantalones blancos, pañoletas al cuello, todo coronado con diversos sombreros ${ }^{3}$. Se hace 
relevante tomar en consideración el impacto visual generado por las naves, acompañado de los objetos, sus colores y gran cantidad de telas que envolvían tanto a personas como a los maderos. Respecto a las reacciones mutuas que se dieron en este primer encuentro, hay que destacar primero las reacciones de los isleños, previo al desembarco de los holandeses. En la relación de Behrens leemos:

\begin{abstract}
Nos trajeron pollos crudos y asados y muchos tubérculos en tierra corrían e iban de un lugar a otro, como bestias salvajes. Grupos de 50, 100 personas se sentaron en la orilla y observaban con asombro nuestros buques, aparentemente curiosos de la novedad del espectáculo o para saber qué habíamos venido a buscar. También alumbraron fuegos frente de sus ídolos, para hacerles ofrendas o bien para implorarlos (Behrens, 1737, pp. 82-83)4.
\end{abstract}

Si bien la narrativa de Behrens es completamente especulativa sobre la razón de las acciones de los rapanui, nos entrega alguna información interesante. Los ídolos a los cuales Behrens hace referencia son los moai, frente a los cuales los rapanui realizaron actividades hoy difíciles de reconstruir, que podrían haber sido acciones rituales ante la llegada de los extranjeros. Argumentamos esto pues en dos de las siguientes expediciones se registran actividades ceremoniales delante de los ahu-moai, las cuales, según las descripciones, se asemejan a los koro paina, fiestas celebradas en honor a un antepasado (Métraux, 1970; Seelenfreund \& Ramírez, 2013).

Un elemento que se encuentra en todas las relaciones es la curiosidad que despiertan la construcción y la envergadura de los barcos europeos, como, por ejemplo, en el caso del primer isleño que hace contacto con ellos y que fue subido a bordo del navío Arend:
En la mañana el Capitán Bouman (...) llevó a nuestra nave un pascuense con su embarcación (...) Esta pobre persona apareció estar muy contenta de vernos, y se maravilló mucho de la construcción de nuestro barco, y lo que el observó de éste, como la gran altura de los mástiles, el grosor de los cabos, las velas, el cañón, el que manipuló cuidadosamente y así todo de lo que vio (Foerster, 2012, p. 78) ${ }^{5}$.

Este primer isleño que sube a bordo realiza una serie de acciones frente a la tripulación, acciones que nos hablan de una forma particular de relacionarse con los extranjeros:

(...) Debió haber estado avergonzado de su desnudez ya que vio que todos estábamos vestidos. Luego puso sus brazos y su cabeza en la mesa, parecía estar dirigiéndose a su dios, lo que podíamos advertir claramente de sus movimientos cuando elevaba su cabeza y manos muchas veces hacia el cielo, usando muchas palabras en voz alta. Atamos una pequeña pieza de lona de vela sobre su área pélvica lo que le agradó de sobremanera. Tenía por naturaleza un semblante feliz (Foerster, 2012, pp. 86-87, énfasis añadido) ${ }^{6}$.

Le hicimos señas de subir a bordo de nuestra nave Almirante, donde lo recibimos bien. Empezamos por darle un trozo de tela con el fin que se cubriera, pues estaba completamente desnudo (...) En seguida le vestimos y le colocamos un sombrero, pero bien vimos que no tenía costumbre pues se sintió muy molesto. (...), lo enviamos a su casa con todos los pequeños presentes, con el fin de que los demás pudieran saber de qué manera lo habíamos recibido. Pero pareció lamentar la partida: levantó ambas manos, volvió los ojos hacia la isla y comenzó a gritar con fuerza pronunciando estas palabras: ¡Odorroga! ¡Odorroga! Le costó bastante decidirse a volver a su canoa, nos hizo entender que él deseaba permanecer en el barco y posteriormente lo desembarcáramos en su isla (Foerster, 2012, p. 72, énfasis añadido) ${ }^{7}$.

Este primer isleño que se acerca al navío es identificado por Fischer (2006) como un tumu ivi 'atua, sacerdote, quien se habría 
aproximado para levantar el tapu (tabú) de los extranjeros, antes del desembarco y hacer los rituales apropiados. Fischer señala, además, que odorroga equivaldría a otoroka en la lengua rapanui antigua, que significa bienvenidos. Lo primero que llama la atención en las relaciones de Behrens y Bouman es que este rapanui llega completamente desnudo. Como vimos anteriormente, el acto de desnudarse era señal de respeto hacia un extraño que es considerado superior (Jolly \& Tcherkézoff, 2009). Aunque el isleño no se desnuda frente a los extranjeros, como en otras islas de la Polinesia, sino que llega ya desnudo al barco, podríamos interpretar este hecho como un acto de respeto hacia los visitantes extranjeros, quienes además le entregaron una pieza de tela. Es más, lo envolvieron en ella. A la luz de estos antecedentes podemos entender dichos gestos como acciones de una ceremonia de recibimiento.

Luego de dos días, el mismo rapanui vuelve a interactuar con los holandeses, pero esta vez se acerca a los barcos, acompañado de otros hombres que traen presentes en forma de comida previamente preparada:

(...) el insular que recibimos dos días antes, vino una segunda vez acompañado de varias personas para traernos gran cantidad de gallinas y raíces preparadas con condimentos a su manera. Entre ellos había un hombre decididamente blanco, llevaba pendientes de orejas blancos y redondos, gruesos como un puño. Tenía un aire extremadamente devoto y la apariencia de uno de sus sacerdotes (Foerster, 2012, p. 72, énfasis añadido) ${ }^{8}$.

Este hombre "decididamente blanco" que Behrens asocia a un sacerdote podría corresponder, debido a la blancura de su piel, a un alto jefe o sacerdote, quien se acerca al barco para iniciar la ceremonia de bienvenida con comida, la cual se ve interrumpida por uno de los hechos que marcará la expedición: Behrens nos relata que al momento de los intercambios uno de los tripulantes disparó contra uno de los pascuenses ${ }^{9}$. Este suceso les otorgará otro elemento de interpretación a los isleños sobre la cualidad de los visitantes, su capacidad de producir truenos y relámpagos, que son capaces incluso de matar a una persona. Truenos y relámpagos en la Polinesia eran considerados pertenecientes al ámbito de lo divino (Tcherkézoff, 2004).

Luego, durante el desembarco, nuevamente sucede un episodio de violencia. Mientras se formaba la tropa adelante, en la retaguardia uno de los suboficiales disparó contra los rapanui que habían llegado a las playas:

\section{Llegamos a la orilla sin resistencia alguna (...) los habi- tantes no tenían armas en lo absoluto, se acercaron a nosotros con las manos vacías para recibirnos, brin- cando y saltando de alegría. A pesar de esto, 9 a 10 de ellos fueron muertos por disparo y muchos heridos. Lo que fue responsabilidad de mi segundo timonel, Cornelis Mens, quien había disparado sin instrucción (Foerster, 2012, pp. 88-89) ${ }^{10}$.}

Las relaciones son contradictorias en cuanto a los hechos de violencia. Bouman, Roggeveen y Behrens (Foerster 2012) señalan que los disparos fueron accidentales, mientras que el anónimo describe una escena donde los rapanui les amanazaban con sus gestos y piedras, razón por la cual se les disparó. Como consecuencia, la población rapanui se dispersó, alejándose de los holandeses.

Luego de un tiempo uno de los jefes regresa y hace las ofrendas necesarias para el apaciguamiento de los extranjeros. En primer lugar, como relata Behrens, hay un ritual que involucra hojas de palma y un estandarte rojo y blanco: 
Todos hombres, mujeres y niños pasando delante de nosotros portaban ramas de palma y una especie de estandarte rojo y blanco. Sus presentes consistían en higos de India, nueces, caña de azúcar, pollos y raíces. Luego se arrodillaron, plantaron sus banderas y nos presentaron sus ramas de palma en señal de paz. Nos dieron testimonio a través de sus humildes posturas de cuanto deseaban nuestra amistad (...) Finalmente nos señalaron sus mujeres, dándonos a entender que podríamos disponer de ellas llevándonos algunas a nuestros barcos (Foerster, 2012, p. 73) ${ }^{11}$.

No sabemos si efectivamente se trataría de hojas de palma, dado que no está claro si en esa época aún quedaban palmeras en la isla. Alternativamente, si no fuesen hojas de palma, podría haberse tratado de hojas de banano. Las hojas de plátano en otras islas del Pacífico se usaban en contextos ceremoniales, como signos de intenciones pacíficas. Además, los estandartes con banderines de color rojo eran usados en momentos de firmar acuerdos de paz después de una guerra (Salmond, 2009). No creemos que este hecho de violencia haya iniciado las relaciones de comercio, como indica Foerster (2012), pues lo que ocurre a continuación es un acto ceremonial, donde las ofrendas indican respeto y sumisión, no intercambio con fines comerciales o económicos:

Un habitante se nos acercó con pasos débiles. Portaba un tocado de plumas blancas en su cabeza, un vestido blanco alrededor de su cuerpo y una concha blanca en su pecho. Ya desde cierta distancia hizo reverencias mediante inclinaciones y otros movimientos de su cuerpo; traía un pollo y un racimo de bananas que puso en el suelo a alrededor de 30 pasos de nosotros y luego huyó como Pegaso. Pero volvió cuando vio que era agradable para nosotros junto con algunos otros habitantes de nuevo trajeron pollos, ñames, bananas, plantas jóvenes y caña de azúcar; le hicimos dejar esto en el suelo y sólo tomamos los pollos y las bananas con nosotros. Este indígena con las plumas era su jefe como pudimos juzgar por aquel evento (...) Al ver lo pobres que eran los honramos en recompensa con una media pieza de tela de Haarlemme ${ }^{12}$ de 5 a 6 peniques por vara, la que aceptaron con gran gratitud (Foerster 2012, pp. 89-90, énfasis añadido) ${ }^{13}$.

(...) nosotros le hicimos entender con signos que queríamos nada más excepto las aves, siendo alrededor de 60 el número y 30 bultos de plátanos para los cuales pagamos el valor generosamente con tela estriada, con cual parecieron estar bastante contentos y satisfechos (Foerster 2012, p. 80, énfasis añadido) ${ }^{14}$.

Los alimentos que se ofrecen tampoco son cualquier alimento, sino los reservados para la élite: pollos, bananos, ñame. No se presentan ratones, que conformaban la dieta del común de la población (Métraux, 1941). Los europeos, a su vez, refuerzan la percepción rapanui, dando a entender su posición sobrenatural al elegir entre los alimentos solo los pollos (generalmente reservados para los ariki y altos sacerdotes) y las cabezas de bananos, despreciando todo el resto de los alimentos, dando cuenta además de que conocen las "reglas del juego" al retribuir las ofrendas de alimentos con los objetos correctos: telas.

Otro elemento que está presente es el hecho de la desnudez femenina y el ofrecimiento sexual que, como vimos anteriormente para el caso de otras islas de la Polinesia, está relacionado con el interés de captar la potencia de los extranjeros:

Sus mujeres están en general maquilladas de un rojo muy vivo y que sobrepasa de lejos el que conocemos (...) Ellas se cubren con coberturas rojas y blancas y llevan un pequeño sombrero hecho de cañas o de pajas. Se sentaban a menudo cerca de nosotros y se desnudaban, sonriendo y provocándonos con toda clase de gestos. Otras que permanecían en sus casas, nos llamaban y nos hacían señas para ir donde estaban (...) (Foerster, 2012, p. 74, énfasis añadido $)^{15}$. 
Pero, al igual que en otras materias, se observan ciertas contradicciones con respecto al tema de las mujeres en los relatos contenidos en las diferentes relaciones. Roggeveen señala que no vieron más de dos o tres mujeres mayores, "pero las mujeres e hijas jóvenes no se mostraron" (Foerster, 2012 p. 81).

Finalmente, parece interesante destacar la dirección desde donde llegaron los holandeses. Al mirar la carta de navegación en el diario de Bouman, vemos que se aproximaron a la isla desde el sureste, dirección de la salida del sol, y se dirigieron hacia el poniente rodeando la isla para luego tomar la ruta hacia el norte, hacia el territorio de los Miru, donde finalmente se hace el desembarco. El hecho de que los holandeses hayan desembarcado en el territorio de los Miru (la tribu de mayor rango) y no en el de otro mata debe haber tenido algún tipo de impacto en la forma en cómo fueron percibidos estos primeros visitantes. Podemos especular que este es uno de los factores que incidieron en que los rapanui identificaran a los europeos como ancestros deificados del clan de Hotu a Matu'a, el mítico jefe fundador, sin embargo, no contamos con suficientes elementos que nos permitan asegurarlo.

Podemos ver, entonces, que, ante esta primera visita de los europeos, la reacción de los rapanui fue recibir a los extranjeros con las ceremonias de bienvenida, que implicaban discursos y ofrendas de comida con la presentación de ramas verdes. Estos rituales, como ha demostrado Pearson (1969), son parte de una recepción formal panpolinesia para los viajeros. Por otro lado, estos quedaron sorprendidos por la reacción de los isleños con actos de sumisión frente los actos de violencia padecidos.
Cabe hacer notar que los hechos descritos en los diarios de viaje de Roggeveen se repiten unas semanas más tarde en forma casi idéntica en Samoa a la llegada de esta misma expedición.-

\section{La expedición española}

La segunda visita a la isla por parte de una nave europea fue casi cincuenta años después. A diferencia de la expedición holandesa, la expedición española al mando del capitán Felipe González de Haedo perseguía un fin colonial. Así, en el año 1770 toma posesión de la isla en el nombre del rey Carlos III de España, bautizándola como isla de San Carlos. Su estadía fue más larga.

En este encuentro observamos que se repiten algunos de los acontecimientos que se desarrollaron durante la visita de los holandeses, pero algunas de las actitudes de los isleños cambian.

\section{Entraron a bordo de ambos buques multitud de yndios de ambos sexos, en quienes siempre se esperimenta mucha sinceridad y Amor; trajeron Platanos, pollos, y raízes, ofreciendo con ynstancia el miserable Ropaje que tienen, que es un misero taparrabo de pita o de ojas de cañas, y una especie de diadema o corona de plumas de Gallo o yerba seca del Mar (Mellén Blanco, 1986, p. 307, énfasis añadido) ${ }^{16}$.}

Lo que primero se observa es que los hombres que van al encuentro de los barcos nadando llevan expresamente comida para entregar a los extranjeros. Se repite, de esta manera, una bienvenida con ofrendas en forma de alimentos. Sin embargo, no encontramos la presencia de un sacerdote o tumu ivi'atua como en el caso de los holandeses, que se acerque solo y desnudo a recibir a los visitantes. En esta ocasión, según 
la relación de Aguera, llegaron varios isleños a bordo de los navíos, lo cual podría estar dando cuenta de la ausencia de una actividad ritual formal de bienvenida.

Este acto resulta importante de destacar, pues evidenciaría una actitud abierta hacia los extranjeros, y no una de rechazo teniendo en cuenta la experiencia de violencia que habían experimentado décadas atrás con los holandeses.

Un hecho que encontramos nuevamente es el recibimiento femenino que los europeos ven como hospitalidad sexual.

Son así como los hombres importunas en el pedir, pero unos y otros ofrezen con la misma franqueza quanto tienen $y$ en particular las mugeres llegan a ofrezer con instancia y demostraciones quanto una de estas tiene que tributar á un hombre apasionado de ellas: Sin que esto al parezer sea reparable respecto de que los mismos hombres las presenten en ofrenda por vía de agasajo (...) advirtiendo que los viejos y mayores tienen alguna preferencia en este asunto, pues siempre estos son los que las acompañan, $y$ ofrecen y a quienes ellas ofrecen subordinación (Mellén Blanco, 1986, p. 284, énfasis añadido) ${ }^{17}$.

Este relato de Aguera nos habla de un hecho que se había dado también en la expedición anterior: el "ofrecimiento" sexual de mujeres a los extranjeros. Sin embargo, aparece un elemento nuevo, esta vez son también los hombres quienes las incitaban a ello, y no todos los hombres, sino que los más viejos. En este sentido, nos encontramos con un paralelo de los sucesos ocurridos en otras islas de la Polinesia, donde las mujeres jóvenes, sobre todo las vírgenes, eran entregadas para capturar el poder de los extranjeros a partir del establecimiento metafórico de un matrimonio sagrado.

Al segundo día un grupo de isleños sube al barco con ofrendas de alimentos: plátanos, tubérculos, pollos y trozos de tela de corteza que a los españoles les parecieron bastante míseros.

Saltamos todos en tierra para comer lo que trahiamos para este fin y vinieron a vernos como 100 Ysleños, ofreciendonos ya Fruta, ya Gallinas. El Oficial Don Cayetano de Langara mandó so pena de ser castigados severamente á nuestra gente, que nadie reciviese cosa alguna de los Ysleños, sin que se les diese otra cosa equivalente (...) pues se conocia que el animo de ellos era de cambiar con otras cosas nuestras y en efecto así se executó (Mellén Blanco, 1986, p. 261)18.

Acá se evidencia una actitud explícita de parte de los europeos de "comerciar" con los isleños, dando cuenta de la importancia que tenía para los españoles establecer relaciones pacíficas satisfaciendo las necesidades de intercambiar que según ellos tenían los isleños.

Aguera nos relata que cuando descienden de los barcos son recibidos por un gran grupo de personas, todas con capas amarillas o blancas sobre los hombros. En el día del desembarco, cuando los españoles iban a tomar posesión de la isla mediante el acto simbólico de posicionar tres cruces sobre los montículos de la península del Poike, sucede un hecho relatado por Hervé que resulta importante destacar: "Al saltar a tierra los recivieron mucha gente de este pais y se ofrecieron á desembarcar á nuestros Oficiales; en efecto así lo executaron, y cargaron las tres cruces, las que llevaron á dichos cerros (...)" (Mellén Blanco, 1986, p 262). Aguera en su diario también describe ese momento:

Luego que las embarcaciones regresaron de la primera expedición salimos nosotros con la segunda, guardan/ do la misma formalidad acompañando las 3 cruces con Bandera desplegada y batiendo marcha: Assi llegamos y con el mejor horden (...) fuimos recibidos de una multitud considerable de Yndios que ayudaron a sacar en hombros nuestra gente a tierra (Mellén Blanco, 1986, p. 309). 
El hecho de querer desembarcar y cargar a los oficiales hasta la orilla da cuenta de la comprensión por parte de los rapanui del estatus de dicho extranjero en relación con el resto de la compañía. Los rapanui identifican a los oficiales y los diferencian del resto del grupo. El hecho de levantar a una persona y cargarla está asociado a la figura del ariki, el cual, en ocasiones, era llevado en andas, pues no debía tocar el suelo a fin de no contaminarlo con su mana y hacerlo tapu para el resto de la población (Métraux, 1941).

González de Haedo y Francisco Aguera nos dejan un informe detallado de las maniobras del desembarco y de la formalidad que ello implicaba. Al igual que los holandeses, la toma de posesión de la isla en nombre del rey Carlos III fue un acto que siguió un protocolo formal en el cual participaron la tropa y la gente de mar, 250 hombres en total, 60 de tropa y 190 hombres de mar, formados en columnas precedidos por los artilleros del Real Cuerpo de Brigadas. En el ascenso hacia los tres cerros para colocar las tres cruces iban además los capellanes. Los rapanui simultánea y activamente celebraban su propia ceremonia, cargando las cruces durante todo el ascenso:

Observé que el día de la colocazion de las Cruzes en que nuestros Capellanes iban acompañando las Sagradas figuras, revestidos de sotana y Pelliz cantando las Letanias les salían al Camino muchos Yndios, ofreciendoles sus mantas, y las mugeres les portavan gallinas y pollos, y todos gritaban Máca Máca, tratandoles con mucha venerazion, hasta adelantarse a quitarles las piedras que embarazavan la senda por donde hivan (Mellén Blanco, 1986, p. 285, énfasis añadido) ${ }^{19}$.

Formada la Tropa y gente de Armas emprehendimos la marcha acompañados de los Yndios quienes ayudaron gustosos a llevar las Cruzes cantando y baylando a su modo: Transitamos todo el circuito de la ensenada con bastante trabajo, por ser el terreno (aunque llano) mui fragoso, llevando siempre al rededor una gran comitiva de naturales hasta el pie del Monte, en donde se quedó la mayor parte por ser la suvida mui penosa, y dilatada. A la 1 1/2 llegamos al sitio destinado para la colocación lo que se celebró a todo juvilo, prezediendo la bendicion, y adoracion de todo el concurso a las Sagradas Imagenes, con cuio exemplo los Yndios practicaron la misma ceremonia. Plantadas ya las Cruzes en sus respectivos cerros se arboló la Vandera española y puesta la tropa sobre las Armas, el Capitán de Fragata Don Josef Bustillos, con las ceremonias acostumbradas tomó posesión de esta Ysla de San Carlos (Mellén Blanco, 1986, pp. 287-288) $)^{20}$.

En el relato de Aguera sobre los acontecimientos nos encontramos con dos hechos destacables en relación con la participación de los rapanui en la toma de posesión del territorio. En primer lugar, cuando los españoles descienden con sus cruces, varios isleños (multitud de ellos) los rodean y los acompañan. Sin embargo, cuando suben el cerro solo algunos los siguen, quedándose la mayor parte al pie de este. Esto nos hace pensar en la significancia del territorio al cual se estaba ingresando. Datos arqueológicos nos indican que cerca de los tres cerritos se localiza un asentamiento habitacional que, según datos etnográficos, estaría asociado a una localidad habitada por sacerdotes especialistas (Vargas, Cristino \& Izaurieta, 2006). Allí también se encuentra la cueva de los Hue Neru, en la que niños y niñas eran recluidos ritualmente para blanquear su piel. Según algunos datos las niñas estaban reservadas para ser casadas con los ariki o sacerdotes. Por lo tanto, los que acompañaron a los españoles hasta los tres domos posiblemente eran sacerdotes o personas de alto rango y el territorio habría estado bajo tapu para el común de la población. En segundo lugar, quienes subieron con los españoles y los acompañaron en las acciones rituales occidentales tuvieron una actitud, como 
señala Foerster (2012), mimética al copiar las acciones rituales de los europeos.

Otro hecho que resulta importante resaltar es la ceremonia que se efectúa el día anterior a la colocación de las cruces, pues no solo muestra la importancia que le otorgaron los rapanui a estos extranjeros, sino que sugiere la clasificación de estos, dentro de la estructura de significación polinésica, como seres con características diferentes a las humanas:

También se le há visto otro ídolo, como de 3 varas, con su cavellara, todo blanco, á que llaman Gecopeca y la mañana del dia antes de la colocación de las Cruces, lo situaron á la orilla del mar, poniendo con orden distintas piedras unas sobre otras, y al rededor se sentaron varios Ysleños (lo que practican en cuclillas como los Moros), permaneciendo en esta forma hasta que con la oscuridad de la noche no fue posible continuar la observación (Mellén Blanco, 1986, p. 274)²1.

Aguera describe esta misma figura, especie de muñeco con extremidades móviles, de unas cuatro varas de alto, que se asemeja a lo que denomina una figura de Judas, rellena con pasto seco. Relata que tenía brazos y piernas y en su cara estaban indicados los ojos, la nariz y la boca. Llevaba pelo negro y largo que le alcanzaba hasta la mitad del dorso. Según comprendió en determinadas ocasiones, estas figuras eran trasladadas y levantadas en los lugares de ceremonia. Dieciséis años más tarde la expedición francesa también tuvo la oportunidad de observar los restos de una de estas figuras de junco levantada frente a una plataforma o ahu, de algo más de 3 metros de altura, cubierta de una tela de corteza blanca (Foerster, 2012) ${ }^{22}$. Tanto Métraux (1941) como Englert (1974) recogieron algunos datos sobre estas figuras y las relacionaron con la celebración de los koro riu paina ${ }^{23}$, acciones rituales ofrecidas a un familiar fallecido.
La ubicación de estas figuras cerca de la línea de costa, frente al ahu, como también el acto de envolver en telas a los muertos o a las figuras que los representan en las celebraciones rituales del koro riu, estarían dando cuenta de una forma particular de entender a los extranjeros. Esta figura levantada frente al barco de los españoles, ¿tenía la intención de dar la bienvenida a los seres ancestrales o fue una ceremonia con la esperanza de mandarlos de regreso a su lugar de origen en el universo, o tal vez un ritual para domesticar sus poderes sagrados? Los españoles llegaron, además, el 5 de noviembre de 1770, en la víspera de la fiesta del Matariki24 . Podría ser que la figura tuviera relación con esta festividad (Edwards y Edwards, 2013).

Los rapanui confrontaron la llegada de los españoles con actos rituales propios. Todo acto ritual persigue un fin determinado. En el caso polinésico se lleva a cabo para poder domesticar las fuerzas invisibles y hacerlas propias. Con ello comienza una manera de actuar que va ser propia de los rapanui al relacionarse con los occidentales: la imitación de las acciones y formas extranjeras.

\section{La visita del capitán James Cook}

Las expediciones inglesas del capitán Cook por los mares del sur fueron de gran importancia para la construcción del imaginario europeo sobre los isleños. Las publicaciones de los diarios de James Cook y de George Forster sobre la segunda de estas expediciones alrededor del mundo (1772-1775) marcaron profundamente a la sociedad europea de la época y dieron forma a la visión que esta tuvo de los pueblos visitados por los viajeros. Fue 
uno de los hitos que marcaron la visión europea sobre las sociedades de los mares del sur.

La visita del capitán Cook a Rapa Nui tuvo ciertas diferencias con las expediciones anteriores. En primer lugar, Cook había acumulado experiencia durante su primer viaje alrededor del mundo (1768-1771) en cómo relacionarse con los "nativos" de otras islas del Pacífico sur. Había estado en las islas de la Sociedad, Nueva Zelandia y Tonga. Como bien comenta Foerster (2012), los fines de la visita de Cook a Rapa Nui estaban relacionados con el suministro de alimentos, para reabastecer sus barcos y, principalmente, para que la tripulación se pudiera recuperar del escorbuto que los aquejaba. Ahora bien, en su descripción de la isla constantemente se puede leer la comparación entre Rapa Nui y Tahiti, en la que la primera siempre quedaba en desventaja frente a la segunda en los ámbitos relativos a la riqueza y la abundancia de la tierra.

La expedición deseaba llegar a Tahiti con premura a sabiendas de que allí encontrarían frutas y verduras en cantidad. Hay que precisar que en cada una de sus visitas a Tahiti, Cook siempre llegó durante la temporada de abundancia y por ello siempre fue agasajado con generosidad (Salmond, 2009). Nunca estuvo durante una temporada de escasez o de invierno. En su visita a Rapa Nui hay que considerar que Cook arriba en marzo, a fines del verano, poco antes de finalizar la temporada de abundancia y al final del ciclo ritual anual (Edwards y Edwards, 2013), hecho que también podría incidir en la cantidad de comida con que contaba la población para esa fecha. Por otro lado, se suma a esto el hecho de que la expedición inglesa contaba con conocimiento previo de Rapa Nui a través de las relaciones holan- desas, narraciones cargadas de una mirada positiva sobre la isla, en las que describen tierras fértiles y abundantes en comida, conocimiento que, como plantea más tarde el conde Jean-François de La Pérouse (Foerster, 2012), indujo en la expedición ciertas expectativas que se vieron contrastadas con una realidad al parecer muy diferente.

Leemos en las relaciones de la expedición inglesa cómo se vuelven a repetir algunos de las acciones que ya habíamos observado durante las expediciones anteriores. En primera instancia, los isleños reciben nuevamente a los extranjeros con regalos de alimentos antes del desembarco:

Llegaron a nuestro lado en poco tiempo, habiendo remado muy enérgicamente e inmediatamente clamaron por una cuerda, nombrándola con la misma palabra que los tahitianos. Apenas lanzada la cuerda, cuando le ataron un gran racimo de bananas maduras, haciendo señales para que las subiéramos (...) El Capitán Cook mandó a buscar algunas cintas, a las que ató algunas medallas y cuentas y las bajó a cambio de su obsequio. Parecieron admirarlas mucho, pero se apresuraron hacia la costa con ellas inmediatamente. Al retirarse ataron un pequeño trozo de tela a una línea de pesca que remolcábamos detrás de nosotros, fue recogida inmediatamente y parecía estar hecha de la misma corteza que la tela tahitiana, y de un color amarillo (...) (Forster, 1777, p. 557) 25 .

Aquí nos encontramos nuevamente con dos actos de bienvenida que podrían ser actos rituales. En esta ocasión, quienes llegan a bordo de una canoa son dos hombres que entregan ofrendas en comida, una cabeza de plátanos y luego atan el trozo de tela al barco. Decimos actos rituales por la información que nos entrega el relato de Forster, quien además cuenta que los rapanui envolvieron con tela un objeto de los extranjeros. Estos actos de 
bienvenida están dando cuenta, nuevamente, de una actitud particular de los rapanui frente a los extranjeros. Al parecer estos no eran considerados como seres que había que expulsar rápidamente sino, por el contrario, seres que había que recibir. Este hecho fue captado por la tripulación inglesa y descrito por las relaciones de Johann Reinhold Forster padre y de su hijo George Forster:

Al ver a unos extraños llegar a [la costa de] su isla, no podían saber cuáles eran nuestras intenciones, ni si no íbamos a cometer algún acto violento. $Y$ a pesar de ello, multitudes de ellos llegaron a la orilla, desarmados, y en vez de oponerse a nuestra llegada, consideraron que era mejor ofrecernos los mejores frutos y raíces que su pobre isla genera; en todo momento eran muy hospitalarios y esta pureza de ellos fue tan conmovedora que nos hizo derramar lágrimas de alegría (Jakubowska-Vorbrich, 2014, p. 81) ${ }^{26}$.

En relación con los objetos que los ingleses traían para intercambiar, de nuevo nos encontramos con telas, pero en este caso la tripulación traía mucha tela de corteza de Tahití, la que usó como medio de intercambio:

Las telas tahitianas y europeas eran valoradas en siguiente grado (después de las cáscaras de coco vacías) según el tamaño de las piezas; y los utensilios de fierro tenían un precio inferior. La mayor parte de los nativos que comerciaban con nosotros corrieron al instante con la tela, la cáscara o el clavo que había sido dado a cambio de sus papas (...) la escasez de tela entre ellos era muy grande, la mayoría del pueblo viéndose obligado a ir desnudo; pero esto no les impedía vender la poca tela que tenían a cambio de la Tahiti (Foerster, 2012, p. 182).

Este último relato de George Forster sobre las telas y las formas de vestir isleñas está nuevamente reproduciendo la narrativa de la escasez y la pobreza de la isla, que "obliga a los isleños a ir casi desnudos". Sin embargo, pareciera que no es un tema de escasez lo que prima, pues la población las intercambiaba de igual modo. Según Foerster (2012), esta forma de vestirse, junto al hecho de no mostrar las fuentes de agua dulce, no entregar tanta comida a los extranjeros, estarían dando cuenta de una actitud planeada por parte de los rapanui frente a los visitantes. Este autor plantea que fue una forma de mostrarse para obtener mayores beneficios de los visitantes. Si bien adherimos a sus postulados en lo relativo a que los rapanui fueron adaptando las formas de relacionarse para obtener beneficios de los europeos, respecto de las formas de vestir creemos que la relativa desnudez descrita por los navegantes tiene que ver más con aspectos rituales que con el deseo de dar una imagen de "pobreza", que por lo demás es pobreza solo a ojos de los occidentales.

Otro elemento en relación a las vestimentas y las telas va ser relevado por George y Johann Reinhold Forster:

Tenían mucha curiosidad acerca de nuestras telas; un trapo de una vieja camisa desgarrada siempre era rápidamente aceptada y a cambio nos ofrecían algunas patatas dulces (...). Nuestra ropa, y especialmente nuestros sombreros, suscitaban tal fascinación entre varios miembros de esta nación (que tiene absolutamente nada para protegerse del calor del Sol) que se llevaron dos o tres [de nuestros sombreros], a riesgo de ser heridos por nuestras armas de fuego (Jakubowska-Vorbrich, 2014, p. 77) ${ }^{27}$.

La relación de los Forster nos entrega una información importante: los isleños no solo estaban interesados en las telas que los europeos llevaban para intercambiar, sino también en las prendas que ellos llevaban puestas, sobre todo en los sombreros. De hecho, los sombreros europeos van a ser uno de los elementos más 
requeridos por los rapanui a la llegada de los barcos. Al respecto, unos años después, La Pérouse (Foerster 2012) va hacer reiteradas referencias a los robos de sombreros a los cuales la expedición francesa se vio confrontada.

Nos encontramos luego con la apreciación de los ingleses de la población femenina de la isla, semejante a la de los relatos de las expediciones anteriores, aunque no se hace mención a la participación de los hombres en el "ofrecimiento de favores sexuales".

Cuando los ingleses desembarcaron, y durante el reconocimiento de la isla, fueron acompañados todo el tiempo por un personaje que portaba un bastón con una especie de bandera blanca "que parecía dirigir la multitud" (Foerster, 2012, p. 205) ${ }^{28}$. Con él pudieron pasar por varios distritos de la isla, donde fueron recibidos formalmente por uno de los ariki locales:

Vimos ahora al rey de pie sobre una colina y caminamos hacia él (...) Llevaba un trozo de tela de corteza de morera, revestido con hilos de hierba y teñido de amarillo con cúrcuma; y en su cabeza tenía un gorro de plumas negras y brillantes, al que podrían llamarlo diadema (Foerster, 2012, p. 185, énfasis añadido) ${ }^{29}$.

Es aquí, durante esta excursión a Vaihu que, a consecuencia de un robo de un bolso, se descarga un tiro contra un isleño, ante lo cual un rapanui reacciona efectuando un acto de humildad y sumisión, al sacarse su capa frente a los extranjeros: "La mayoría del resto huyó aunque algunos permanecieron y un hombre sacándose su ah-hou habló muchísimo y después corrió varias veces alrededor de nosotros, tras lo cual ellos desplegaron una vez más su bandera (...)" (Foerster, 2012, p. 206) ${ }^{30}$. Es muy relevante este hecho pues nos encon- tramos con una reacción similar a la narrada durante la visita de la expedición holandesa, en la que los rapanui, frente a las armas de fuego de los extranjeros, van a reaccionar con actos que nos hablan de una actitud de sumisión.

Vemos que, si bien hay cosas que claramente habían cambiado en esos 55 años desde la primera visita de un barco europeo a las costas de Rapa Nui, ciertos elementos se mantienen y se repiten. En cada una de las visitas, los extranjeros europeos son recibidos con actos de bienvenida que incluyen la ofrenda de comida y la participación de las telas. En relación con las mujeres, todas las expediciones hacen referencia a su escasa cantidad en comparación con la población masculina, además de resaltar sus actitudes lascivas y provocadoras.

\section{Conclusiones}

Con el tiempo y la llegada de más barcos, estas visitas inicialmente esporádicas se hicieron parte de la vida de los isleños y se desarrolló una cultura de relaciones entre los barcos y los rapanui con base en el comercio, donde cada lado fijó condiciones y precios, objetos que se valoraban y que también incluía el intercambio sexual, que siguió siendo promovido por los ancianos y las mujeres mayores. Es interesante ver que al igual que en el resto de la Polinesia, las llegadas de las expediciones europeas del siglo XVIII convirtieron un acto ceremonial de bienvenida en el establecimiento del comercio entre rapanui y extranjeros. En este sentido, tal como lo plantea Mauss (2012), el intercambio de dones entre visitantes y rapanui es un operador del vínculo que regula la hostilidad y la convierte en competencia o en rango para posibilitar una relación pacífica o una alianza o bien un pacto social. 
Las formas de entender y utilizar el textil en Rapa Nui dan cuenta de una estructura significativa común al resto de la Polinesia. Para los rapanui, que observaron desde la playa y recibieron a estos extranjeros visitantes, también fue menester incorporarlos al mundo conocido, a un mundo donde los ancestros vienen de más allá del mar, donde la historia en cada isla comienza con las canoas fundacionales. Todos los antepasados que venían de más allá del horizonte y encontraron su respectiva isla se transformaron en atua. Atua se ha traducido como divinidad o algo de carácter divino (Olivier, 1975). En la cosmovisión polinesia, el ser divino no necesariamente representa algo del "otro-mundo"; ser divino también es una característica de "este-mundo", una característica de los humanos, de los ancestros que llegaron primero. Por lo tanto, se asocia a estos extranjeros venidos de más allá del horizonte con los antepasados que también cruzaron el mar en sus canoas, por lo que frente a ellos hay que actuar con cautela, pues están cargados con mucho mana. Esta es la razón por la cual los rituales para acercarse a ellos deben ser los adecuados y propicios.

El rol de los textiles en este contexto, ya sea mediante el uso de banderines, trozos de tela que se amarraban a los barcos o se usaban para tapizar el suelo que pisarían los extranjeros, refuerza la noción de sacralidad y de los poderes que estos ostentan para dar o quitar vidas. En muchas sociedades del Pacífico, el poder de los altos jefes, en particular sus poderes vitales, podía contenerse mediante el uso de los textiles. Estos marcan y aíslan la sacralidad peligrosa de una persona o un lugar cubriéndolos o envolviéndolos. Esto permitía domesticar a los visitantes. Los intercambios de alimentos y textiles en estos primeros encuen- tros estaban, entonces, orientados a capturar el mana al mismo tiempo que se aplacaba o domesticaba a los seres extranjeros. Este intercambio de dones se debe entender a partir de la capacidad simbólica de estos objetos para capturar el vínculo con los orígenes del grupo. Los textiles y los alimentos son los elementos vitales que, ofrecidos como bienes, refuerzan su carácter de objetos que son fuente de vida y que permiten que la vida diaria se desarrolle a través de una espiral de relaciones de intercambio (Salmond, sin fecha).

A la luz de estos antecedentes vemos que el textil dentro de la sociedad rapanui previa al contacto con Occidente tuvo un rol similar al que desempeñaba en los contextos rituales polinésicos. De hecho, Ilama la atención que los isleños se muestren interesados en la adquisición de telas europeas, de sus chaquetas, sombreros y pañuelos. Los europeos, por su lado, no parecen dar importancia a las telas nativas, excepto como elementos para cubrir el cuerpo, pues no están preparados para ver en ellas su capacidad de agencia simbólica. Los textiles simbolizan el asiento o el camino de los dioses sobre la tierra: a través de las telas se les invita a participar en el mundo de los humanos. La colocación de telas de corteza en el camino de los españoles durante su subida a la colocación de las cruces, junto al canto ritual de Maca Maca, subraya la invitación que se les hace a estos dioses-ancestros. Postrarse frente a ellos demuestra su humildad frente al invitado, que es tratado como un superior, en este caso una deidad o un representante de la deidad. La eficacia de este acto se vio aumentada además por las vestimentas que portaban los españoles, en particular las capas de los padres que acompañaban la comitiva española. 
Por parte de los europeos, los regalos de piezas de telas a los nativos se entregan como parte de los bienes que se tenía para el intercambio y que eran considerados simples bagatelas. De hecho, solo George Forster menciona que el tahitiano Mahina, que iba a bordo, obtuvo de los nativos varios tocados de plumas y una mano finamente tallada de madera que hoy se encuentra en el Museo Británico $\left(\right.$ Foerster 2012) ${ }^{31}$. Entre las piezas intercambiadas también se encontraba una capa de tela pespuntada que actualmente se encuentra en el museo Pitt Rivers de Inglaterra, junto con un tocado de plumas y un pendiente de hueso (Coote, 2014). Los españoles no hacen ninguna mención a objetos que podrían haber sido intercambiados con los nativos, a pesar de encontrarse en la colección de objetos del Pacífico del Museo de América en Madrid una manta de tela de corteza bordada con hilvanes que proba- blemente provenga de Isla de Pascua por su semejanza con la capa colectada por los Forster y la capa del Museo Nacional de Historia Natural de Santiago, colectada por Rodulfo Amando Philippi en 1886 (Seelenfreund, 2013). Por tanto, no tenemos mayores detalles respecto a cómo se habría desarrollado el ritual de donación de telas en la isla. Sí llama la atención que, a diferencia de otras islas, las telas eran mucho más escasas y, como mencionan Cook y George Forster (Foerster, 2012), pequeñas y su elaboración al parecer menos fina que las de Tahití. Circunstancialmente, uno de los elementos más cotizados para el intercambio en su visita fueron precisamente las telas. Los textiles fueron objetos mediadores privilegiados en estos intercambios pues tenían la capacidad de articular lo autóctono con lo extranjero, regulando así las fronteras en una situación de contacto cultural. 


\section{Notas}

${ }^{1}$ El concepto mana hace referencia a lo sagrado como una fuerza total que mantiene a los individuos atados al grupo y que siempre está en circulación Mauss (2012). Keesing (1984) señala que para la Polinesia oriental el concepto de mana está relacionado con el poder espiritual del jefe. Su cabeza es el receptáculo del mana de los ancestros, siendo este el medio invisible que conecta al jefe con la divinidad. Por esta razón, la figura del jefe está rodeada de una serie de prohibiciones, particularmente su cabeza, la cual es considerada tapu. En la Polinesia occidental, el mana no se refiere a una sustancia, una energía invisible y espiritual en sí, sino a una potencia que se evidencia en los resultados de una acción. El mana sería una cualidad, un estado que estaría dando cuenta de la eficacia, del éxito de una acción. Así, en el ámbito de lo cotidiano una persona tendría mana si las acciones que emprende son exitosas mientras que en el ámbito de lo sagrado un ritual o un objeto tendría mana en función de que el resultado fuera eficaz (Bowden, 1979; Keesing, 1984).

${ }^{2}$ El concepto de tapu refleja el tabú de lo sagrado; lo sagrado debe ser mantenido bajo ciertas restricciones, alejado y protegido, por ende, está rodeado de una serie de prohibiciones, como no ser tocado, no ser visto, no ser mirado.

${ }^{3}$ Véase láminas $91878,91890,91886$, entre otras, en: https:// on.nypl.org/2CA7kgb (consulta: 11/12/2018).

${ }^{4}$ Traducción propia de: “(...) brachten uns ungekochte und gebratene Hühner, nebst vielen Wirzeln, und am Lande Lieffensie wie das Wild dem Steande auf und nieder: Theis hatten sie by 50. Und hunderten niedergelassen, und sahen unsere Schiffe mit Verwunderung an; Theils wohl aus Curiosität, Theils aber wolten sehen, was wir da Suchen wollten. Bey ihren Götzen legen sie viel Feuer an, um zu opffern oder zu beten".

${ }^{5}$ Bitácora de J. Roggeveen.

${ }^{6}$ Bitácora de C. Bouman.

${ }^{7}$ Memoria de C. F. Behrens.

${ }^{8}$ Memoria de C. F. Behrens.

${ }^{9}$ Este acontecimiento solo se describe en esta relación, está ausente en las otras tres relaciones de la misma expedición (Anónimo, Bouman y Roggeveen).

${ }^{10}$ Bitácora de C. Bouman.

${ }^{11}$ Memoria de C. F. Behrens.

${ }^{12}$ Se refiere a textiles de Haarlem, que era un centro de blanqueado de telas de seda y lino importantes en Holanda en el siglo XVII y XVIII (Crespo Solana, 2006).

${ }^{13}$ Bitácora de C. Bouman.

${ }^{14}$ Bitácora de J. Roggeveen.

${ }^{15}$ Memoria de C. F. Behrens

${ }^{16}$ Diario de navegación de F. A. Agüera Infanzón.

${ }^{17}$ Diario de navegación de F. A. Agüera Infanzón.
${ }^{18}$ Bitácora de J. Hervé.

${ }^{19}$ Diario de navegación de F. A. Agüera Infanzón.

${ }^{20}$ Diario de navegación de F. A. Agüera Infanzón.

${ }^{21}$ Bitácora de González de Haedo.

${ }^{22}$ Bitácora de P. A Marie Fleuriot.

${ }^{23}$ Para una descripción más detallada y una discusión sobre el rol de estas figuras en la antigua cultura rapanui, véase Seelenfreund \& Ramírez 2013, pp. 38-35.

${ }^{24}$ La festividad de Matariki es una celebración panpolinésica que celebra la renovación cósmica. Comienza con la aparición de las Pléyades a mediados de noviembre y finaliza cuando las Pléyades se vuelven a esconder tras el horizonte a mediados del mes de abril. Durante ese tiempo se hacían ceremonias y fiestas en honor a los ancestros y las divinidades patronas de la subsistencia (Edwards y Edwards, 2013, pp. 444-445).

${ }^{25}$ Traducción del original en inglés "They were along side in a short time, having paddle very briskly, and immediately called out for a rope, naming it by the same word as the Tahitians. We had no sooner thrown them a rope, than they tied a great cluster of ripe bananas to it, making signs for us to haul it up (...) Cap. Cook send for some ribbands, to which he toed some medals and beads, and lowered them down in return for their present. They seems to admire them much, but hastened ashore with them immediately. In dropping astern they fastened a small piece of cloth to a fishing-line which we towed after us, it was immediately towed up and appeared to be made of the same bark as the Tahitian cloth, and coloured yellow".

${ }^{26}$ Relato de J. R. y G. Forster. Traducción propia de la versión en inglés del original en francés: "Seeing strangers arriving at [the coast of] their island, they neither could figure out what were our intentions, nor if we were not going to commit some violent act. And in spite of that, crowds of them were coming to the shore, unarmed, and-instead of opposing to our arrival—they found it better to offer us the best fruits and roots that their poor island yielded; all the time they were extremely hospitable and this purity of theirs was so touching that it made us shed tears of joy".

${ }^{27}$ Relato de George Forster. Traducción propia de: "They were extremely curious about our canvas; a rag of an old, torn shirt was always hastily accepted and they offered us some sweet potatoes in return (...) Our clothes, and especially our hats, excited such fascination among several members of this nation (that has absolutely nothing to protect themselves from the heat of the Sun) that they took away two or three [of our hats], at the risk of being wounded by our firearms".

${ }^{28}$ Relato de R. Pickersgill.

${ }^{29}$ Relato de G. Forster.

${ }^{30}$ Relato de R. Pickersgill.

${ }^{31}$ Relato de G. Forster. 


\section{Referencias bibliográficas}

Babadzan, A. (2003). The Gods stripped bare. En Colchester, C. (Ed.), Clothing the Pacific. (pp. 25-50) Oxford, New York: Berg Publishing.

Behrens C.F. (1737). Reise und Begebenheiten durch die bekannte und unbekannte Südländer und um die Welt. Frankfurt: Joachim von Lahnen.

Bowden, R. (1979). Tapu and Mana: ritual authority and political power in traditional Maori society. The Journal of Pacific History, 14(1), 50-61.

Campbell, I.C. (1982). Polynesian perceptions of Europeans in the 18th and 19th centuries. Pacific Studies, 5(2), 64-80. (1997). Culture Contact and Polynesian Identity in the European Age. Journal of World History, 8(1), 29-55. (2003). The culture of culture contact: refractions from

Polynesia. Journal of World History, 14(1), 63-86.

Colchester, Ch. (Ed). (2003). Clothing the Pacific. Oxford, New York: Berg Publishing.

Coote, J. (2014) Prefacio. En, Jakubowska-Vorbrich Z. (Ed.) Still More to Discover. Easter Island in an Unknown Manuscript by the Forsters from the 18th Century / Wciąż odkrywana. Wyspa Wielkanocna w nieznanym rękopisie Forsterów z XVIII wieku. Varsovia: Biblioteka Iberika. Muzeum Historii Polskiego Ruchu Ludowego, e Instytut Studiów lberyjskich i lberoameryka skich. Pp. XIII-XIV.

Crespo, A. (2006). América desde otra frontera: La Guayana holandesa (Surinam) 1680 -1795. Madrid: Consejo Superior de investigaciones científicas, Colección América.

Dening, G. (1980). Islands and Beaches: Discourse on a Silent Land: Marquesas, 1774-1880. Honolulu: The University of Hawaii Press.

Edwards, E. \& Edwards, A. (2013). When the universe was an island. Exploring the cultural and spiritual cosmos of ancient Rapa Nui. Hanga Roa: Hanga Roa Press.

Englert, S. (1974). La Tierra de Hotu Matua. Santiago: Editorial Universitaria.

Fischer, S.R. (2006). Island at the End of the World: The Turbulent History of Easter Island. Chicago: The University of Chicago Press, Reaktion Books.

Flenley, J. (1996). Further evidence of vegetational change on Easter Island. South Pacific Study, 16 (2), 135-141

Flenley, J. R., King, A.S.M., Teller, J.T., Prentice, M.E., Jackson, J. \& Chew, C. (1991). Late quaternary vegetational and climatic history of Easter Island. Journal of Quaternary Science, 6(2), 85-115;

Foerster, R. (2012). Rapa Nui, primeras expediciones europeas. La construcción dialógica de la Isla de Pascua (Siglo XVIII). Rapa Nui: Rapanui Press.

Forster, G. (1777). A Voyage around the World in his Britannic Majesty's Sloop, Resolution commanded by Capt. James Cook, during the Years 1772, 3, 4 and 5. Vol. 1 Londres. B White, J Robson, P. Elmly y G. Robinson, impresores.
Horrocks, M., Baisden, W.T., Nieuwoudt, M.K., Flenley, J., Feek, D., González Nualart, L., Haoa-Cardinali, S. \& Edmunds Gorman, T. (2012). Microfossils of Polynesian cultigens in lake sediment cores from Rano Kau, Easter Island, Journal of Paleolimnology, 4(2), 185-204.

Howe, K. (1977). The fate of the Savage in Pacific Historiography. New Zealand Journal of History, 11(2), 137 - 154.

Jakubowska-Vorbrich, Z. (2014). Still More to Discover. Easter Island in an Unknown Manuscript by the Forsters from the 18th Century / Wciąż odkrywana. Wyspa Wielkanocna w nieznanym rękopisie Forsterów z XVIII wieku. Varsovia: Biblioteka Iberika. Muzeum Historii Polskiego Ruchu Ludowego, e Instytut Studiów Iberyjskich i lberoameryka skich.

Jolly, M. (2014). A Saturated History of Christianity and cloth in Oceania. En Choi, H \& Jolly, M (Eds.). Divine Domesticities: Christian paradoxes in Asia and the Pacific (pp. 429-454). Canberra: ANU Press, The Australian National University.

Jolly M. \& Tcherkézoff, S. (2009). Oceanic Encounters: A Prelude. En Tcherkézoff, S. (Ed) Oceanic Encounters: exchange, desire, violence (pp. 1-36). Canberra: ANU E Press. The Australian National University.

Keesing, R.M. (1984). Rethinking "Mana". Journal of Anthropological Research, 40(1), 137-156.

Küchler, S. (2003). The Poncho and the Quilt: Material Christianity in the Cook Islands. En Colchester, C. (Ed.), Clothing the Pacific (pp. 97-116). Oxford. New York: Berg Publishing.

McLachlan, S. (1982). Savage Island or savage history? An interpretation of early European contact with Niue. Pacific Studies, 6(1), 26-51.

Mauss, M. (2012). Ensayo sobre el don: Forma y función del intercambio en las sociedades arcaicas. Madrid: Katz editores.

McCall, G. (1990). Rapanui and outsiders: The early day. En Bruno Illius \& Matthias Laubscher (Eds.), Circumpacifica. Festschrift für Thomas S. Barthel (pp. 165-225). Frankfurt am Main: Peter Lang.

Mellén Blanco, F. (1986). Manuscritos y documentos españoles para la historia de la Isla de Pascua. Madrid: CEDEX.

Métraux, A. (1941). La Isla de Pascua. Barcelona: Editorial Laertes. (1970). Ethnology of Easter Island, Bernice P. Bishop Museum Bulletin 160, Honolulu: Bishop Museum Press.

Olivier, D. (1975). Ancient Tahitian Society. Honolulu, University of Hawaii Press.

Pearson, W.H. (1969). European Intimidation and the Myth of Tahiti, Journal of Pacific History, 4(1), 199-21.

Quanchi, M. \& Adams, R. (1993). Culture Contact in the Pacific: Essays on Contact, Encounter and Response. Cambridge: Cambridge University Press.

Sahlins, M. (1997). Islas de Historia: La muerte del Capitán Cook, metáfora, antropología e historia. Barcelona: Ed Gedisa.

Salmond, A. (2009). Aphrodite's Island: The European Discovery 
of Tahiti. Auckland: Penguin Books.

(sin fecha). HAU: Journal of Ethnographic Theory. https:// www.haujournal.org/index.php/hau/pages/view/endorsements (fecha de acceso 15.04.2018).

Seelenfreund, A. \& Ramírez, F. (2013) El universo textil de Rapa Nui. En Seelenfreund, A. (Ed.) Vistiendo Rapa Nui: Textiles vegetales, Haka'ara o the kahu (pp. 38-35). Santiago: Editorial Pehuén.

Seelenfreund, A. (Ed.) (2013). Vistiendo Rapa Nui: Textiles vegetales, Haka'ara o the kahu. Santiago: Editorial Pehuén.

Tapsell, P. (1997). The flight of pareraututu: an investigation of taonga from a tribal perspective. The Journal of the Polynesian Society, 106(4), 323-374.

Tcherkézoff, S. (2003). On Cloth, Gifts and Nudity: Regarding Some European Misunderstandings during Early Encounters in Polynesia. En Colchester, Ch. (Ed.) Clothing the Pacific (pp. 51-75). Oxford, New York: Berg Publishing. (2004). First Contacts in Polynesia. The Samoan Case
(1722-1848). Western Misunderstandings about sexuality and divinity. Nueva Zelandia y Australia: MacMillan Brown Center for Pacific Studies \& The Journal of Pacific History.

(2012). More on Polynesian gift-giving. The Samoan Sau and the fine mats (Taonga), the Maori Hau and the treasures (taonga). Hau: Journal of Ethnographic Theory, 2(2), 313- 324.

Thomas, N. (1991). Entangled Objects: Exchange, Material Culture and Colonialism in the Pacific. Cambridge, MA: Harvard University Press.

(2003). The Case of the Misplaced Ponchos: Speculations Concerning the History of Cloth in Polynesia. En Colchester, Ch. (Ed.) Clothing the Pacific (pp. 79-96). Oxford. New York: Berg Publishing.

Vargas, P., Cristino, C. \& Izaurieta, R. (2006). 1000 años en Rapa Nui. Arqueología del asentamiento. Santiago. Editorial Universitaria S.A.

Weiner, A. (1992). Inalienable possessions: The paradox of keeping-while-giving. Berkeley, University of California Press. 